\title{
MATHEMATICAL MODELLING OF THE ELASTIC PROPERTIES OF RETINA: A DETERMINATION OF YOUNG'S MODULUS
}

\author{
I. L. JONES ${ }^{1}$, M. WARNER ${ }^{1}$, J. D. STEVENS ${ }^{2}$ \\ Cambridge and London
}

\begin{abstract}
SUMMARY
The retina can be regarded as an elastic membrane or sheet which stretches and deforms when a force is applied to it. Isolated bovine retina was taken and a graded traction force applied to determine retinal profile as a function of force. The resulting profile can be modelled mathematically and the model then used to determine a value for the elastic constant. The value of the elastic constant obtained by this method is approximately $2 \mathrm{~N} / \mathrm{m}$. This value of the elastic constant, combined with the observed retinal thickness, yields a value of Young's modulus for retina of approximately $2 \times 10^{4} \mathrm{~Pa}$, which is about 2 orders of magnitude weaker than typical rubber. This value can then be used in modelling retinal behaviour in vivo when forces are applied to detached retina.
\end{abstract}

The retina is a biological membrane that possesses elastic properties and can undergo marked stretching which may be seen in vivo with diabetic traction retinal detachment and traction retinopathy associated with retinopathy of prematurity. ${ }^{1}$ Further clinical evidence of retinal stretching may be seen when retinal blood vessels alter their normal course and become straightened. Work in vitro has also demonstrated that the retina may be stretched and possesses elastic properties, where if free retina is pulled, it stretches until it tears..$^{2-6}$

The neuroretina is a biological membrane which develops from a pseudostratified neuroepithelium where tall cells pass through the full retinal thickness, reaching inner and outer surfaces of the retina. ${ }^{7}$ Later in development, cell differentiation causes the nerve and glial cells to lose their surface connections and the retina develops a layered appearance with horizontally oriented cells and ganglion cells. Photoreceptors become sited with vertical orientation, interconnecting by synaptic processes and glial cells which arborise. This arborising and layered retinal structure is potentially capable of reversible stretching.

From: 'Cavendish Laboratorý, Madingley Road, Cambridge, UK; ${ }^{2}$ Moorfields Eye Hospital, City Road, London, UK.

Correspondence to: Mr. J. D. Stevens, FCOphth, Moorfields Eye Hospital, City Road, London EC1V 2PD, UK.
The retina is approximately $0.1 \mathrm{~mm}$ thick, although its thickness increases to $0.23 \mathrm{~mm}$ in the peripheral macula. ${ }^{8}$ The central fovea is much thinner, but this area is very localised and is surrounded by the thicker peripheral macular tissue.

An in vitro study of retinal elastic properties has been performed by Kain ${ }^{9}$ in which subretinal injection of fluid was administered to assess the shape of the resultant retinal detachment. A consistent finding of a circular retinal detachment was obtained, which was interpreted as the adhesion force between retina and retinal pigment epithelium being equal in all directions. The retina is therefore a thin membrane that has elastic properties and can stretch reversibly.

In a previous publication ${ }^{6}$ we presented a mathematical model of the removal of an epiretinal membrane from the

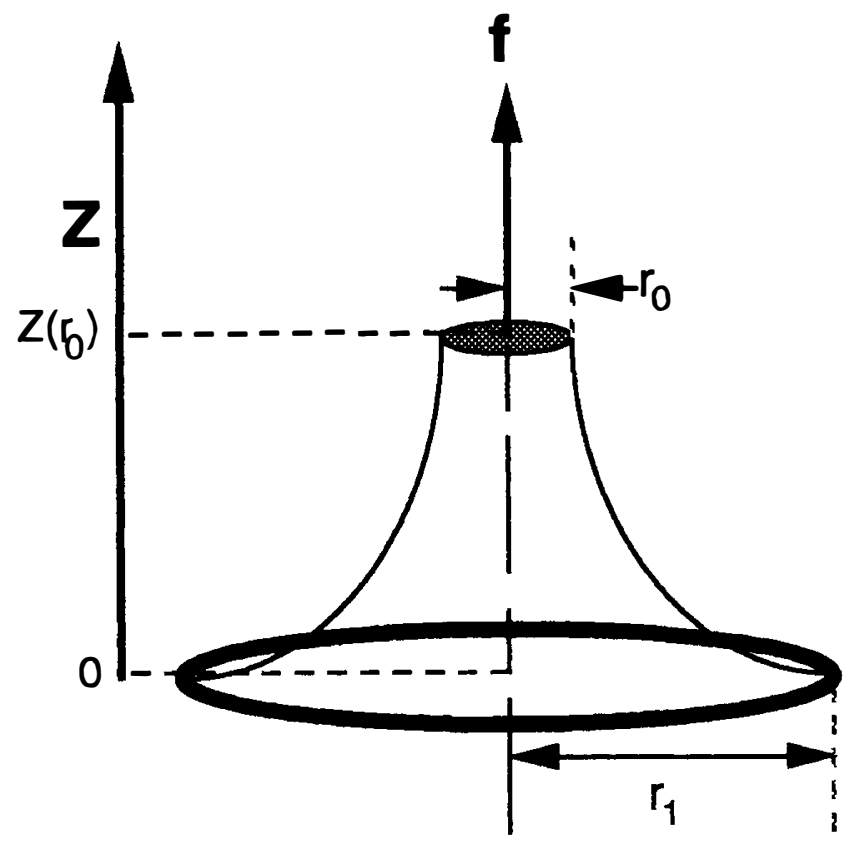

Fig. 1. An idealised retina deforming under the action of a traction force $\mathrm{f}$. The retina is clamped at the outer radius $r_{1}$, and is fixed to a disk of radius $r_{0}$ at its centre. The retina stretches to a height $\mathrm{z}\left(r_{0}\right)$ at the highest point. 


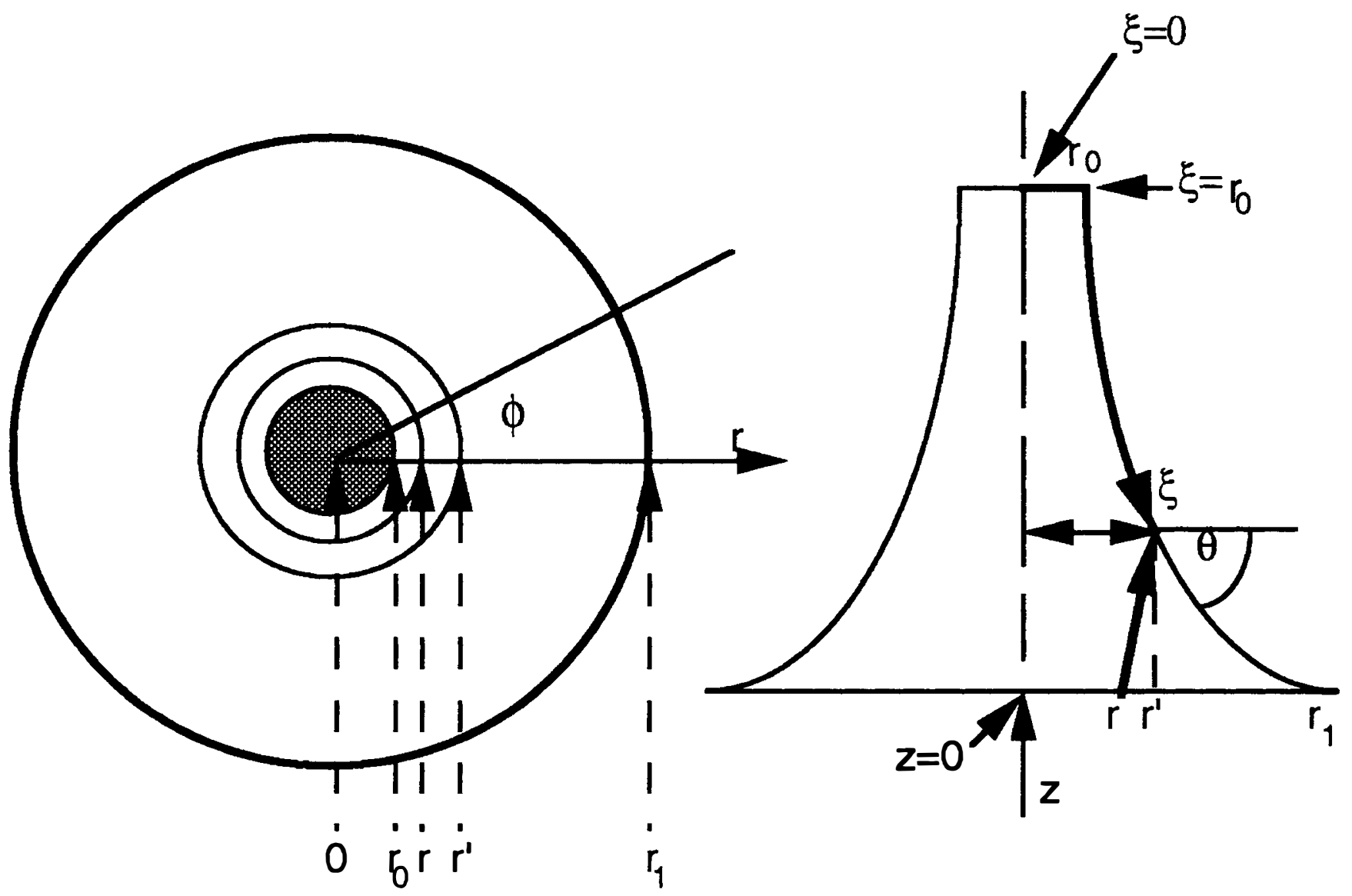

Fig. 2. The coordinate system used to describe the experimental set-up. The independent variable is $\xi$, that is all variables are expressed as functions of $\xi$. The diagram on the left shows a view of the stretched retina from the top viewed along the symmetry axis, and that on the right a side elevation of a central cross-section.

surface of the retina by the application of a pulling force $f$. The retina was treated as an elastic sheet with an elastic constant $k$, and regarded as isotropic and homogeneous within the sheet. We used a simple infinitesimal elasticity model, i.e. a simple Hooke's law relationship assuming the stress in a particular direction is proportional only to the strain in that direction and is, therefore, independent of strains in other directions. This corresponds to a Poisson ratio $\sigma$ of 0 . In this paper we use a more realistic model of $\sigma=1 / 2$, corresponding to a weak incompressible solid. Such solids are volume-preserving, that is the energy scale for compressing the solid, i.e. changing its volume, is typically 3 orders of magnitude greater than the energy scale associated with elastic deformations. The solid can therefore be regarded as incompressible, $\leftrightarrow \sigma=1 / 2$. This has a marked effect on the retinal profiles and resulting induced tensions. We use the model to fit data from the experiments on stretching bovine retina and in this way obtain an estimate for Young's modus $E$ and, by combining with the retinal thickness $\tau$, the elastic constant $k$ for bovine retina.

\section{EXPERIMENTAL METHOD}

Fresh bovine retina is separated from underlying retinal pigment epithelium by peripheral infiltration of Ringer's solution between retina and retinal pigment epithelium. ${ }^{6}$ The retina is gently excised from its attachment to the optic disk and ora serrata by $360^{\circ}$ retinotomy and floated off onto a metal washer of known internal diameter. The metal washer is moved under the free-floating retina so that the macular region and retina adjacent to the optic disk are outside the area contained by the inner diameter of the metal washer. The washer is then elevated to the surface of the Ringer's solution and, on lifting clear of the surface, the retina is spread across the surface of the washer with the inner retinal surface (that facing the vitreous cavity) uppermost. Excess fluid is removed from the retina by the peripheral application of triangular swabs. To maintain a non-slip apposition of the retina to the washer, a ring of butylacrylate (Histoacryl) cement is applied so that the rim of the retina is fixed to the washer. The isolated retina and metal ring are maintained in a humid environment at $20{ }^{\circ} \mathrm{C}$ so that drying out does not occur. It is important to remove the excess moisture from both the upper and lower retinal surfaces to facilitate butylacrylate fixation of the retina to the washer and to prevent the retina sagging unduly in the centre under the weight of excess fluid.

Poorly mounted retinal preparations, or those with visible tears, are discarded. When a successful preparation is made, the retinal disk is mounted in the plane of the retina without sagging at the centre. A 10.0 nylon suture (Ethicon) is suspended from a micro-balance apparatus and a drop of butylacrylate glue run down the thread under 

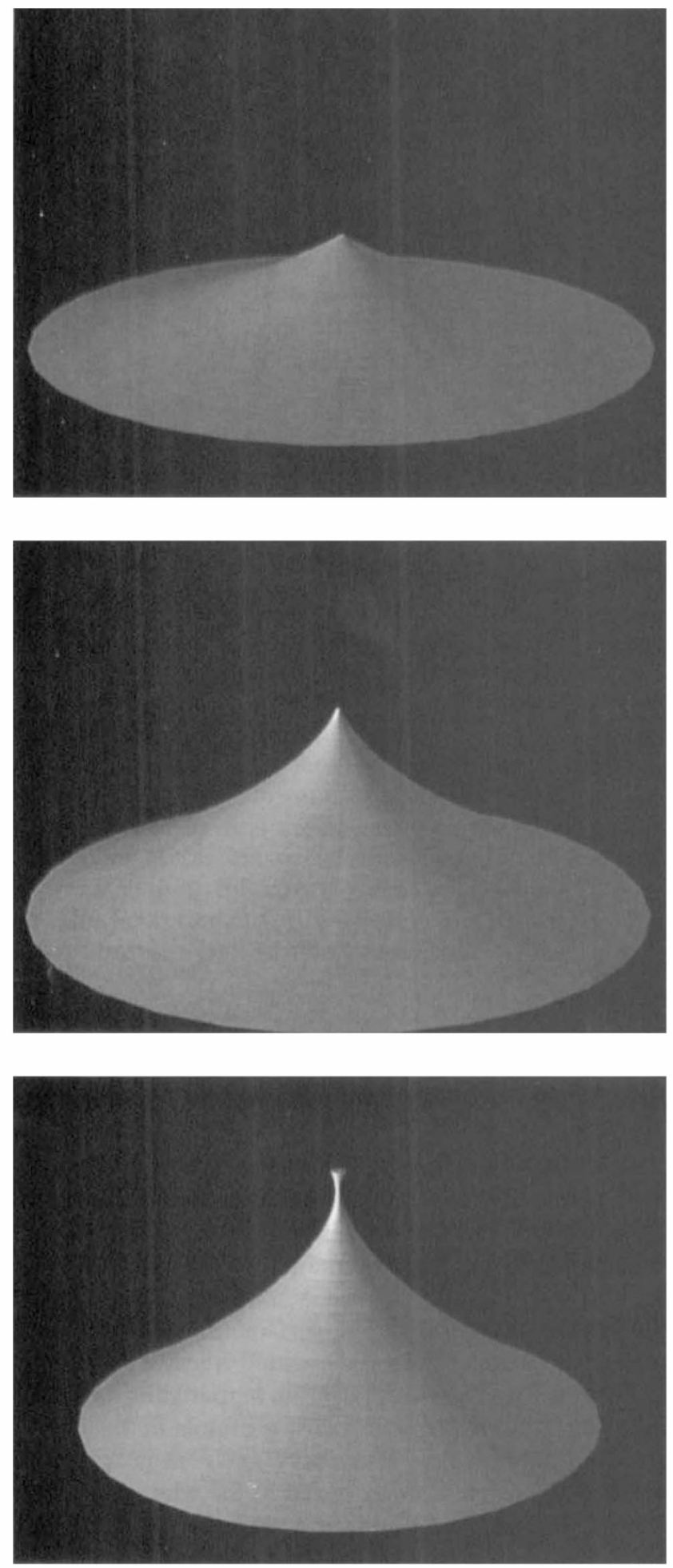

Fig. 3. Typical retinal profiles obtained from solving the equations (1) to (5) subject to the boundary conditions given in the text. The three figures show profiles from an increasing traction force: smallest at the top, largest at the bottom.

its own weight. If the quantity of glue is excessive it is easily removed using a triangular swab and re-application of the glue performed. When application is successful, the small spherule of glue at the lower aspect of the suspended thread is placed in contact with the retinal disk at its centre. The estimated average diameter of the glue attach- ment on the retina in each experiment is taken as $1 \mathrm{~mm}$. After a 2 minute period to allow the glue to bond, graded traction is then applied to the suture. A photographic apparatus records the profiles of the retinas as they undergo stretching. Measurements of the (height of deformation)/ (traction force applied) are taken. Different metal washers are used with a series of known internal diameters.

\section{MATHEMATICAL MODELLING}

To apply a model to the experimental situation described above, we consider the retina to be an elastic sheet of constant thickness $\tau$ and elastic constant $k$ (or Young's modulus $E$.) In what follows, when we refer to retina we mean this idealised retina. The retina is now fixed at a circular boundary, of radius $r_{1}$, and a fixed disk of radius $r_{0}\left(<r_{1}\right)$ is attached to the centre of the retinal disk. A force $f$ is applied at the centre of the small fixed disk in a direction perpendicular to the plane of the unstretched retina. The retina will deform and adopt a shape with a profile similar to that shown in Fig. 1, as seen in the bovine retinas.

To apply a model, we describe the stretched retina using the following coordinate system, which is shown in Fig. 2. Let $r$ be the radial coordinate, measured from the symmetry axis (axial symmetry will be assumed throughout) and $z$ the perpendicular distance from the plane of the unstretched retina. The azimuthal angle is given by $\phi$, measured from some arbitrary radial line.

Consider a small element of retina that before stretching was at the position $(r, \phi, 0)$. After stretching, when the force has been applied, the same element will be found at position $\left(r^{\prime}, \phi, z\right)$. The displacement in the radial direction is $r^{\prime}-r$, in the vertical direction is $z$, and we assume that $\phi$ remains the same, i.e. that there is no twist. We will use as the independent variable $\xi$, which is the 'in-surface' coordinate (i.e. the curvilinear distance from the centre of the small disk along the surface at constant $\phi$ to the element in question). Using $\xi$ as the independent variable, rather than $r^{\prime}$ as we did previously, can avoid singularities in the resulting equations as $\theta$, the angle between the vertical tangent and the starting plane, approaches $\pi / 2$.

The resulting equations to solve are:

$$
\begin{aligned}
& \dot{\eta}=(1-\eta)^{2}\left[\left(\frac{t_{\phi}-t_{\xi}}{k r^{\prime}}-\frac{1}{2} \frac{1}{\xi-p}\right)\right. \\
& \left.\cos \theta+\frac{r^{\prime}}{2} \frac{(1-\eta)}{(\xi-p)^{2}}\right] \\
& \dot{\theta}=\frac{-t_{\phi}}{t_{\xi}} \frac{\sin \theta}{r^{\prime}} \\
& \dot{p}=\eta \\
& \dot{r}^{\prime}=\cos \theta \\
& \dot{z}=\sin \theta
\end{aligned}
$$

where $p(\xi)=\xi-r(\xi) ; \eta=\mathrm{d} p / \mathrm{d} \xi$; and $t_{\xi}$ and $\mathrm{t}_{\phi}$ are tensions (in $N / \mathrm{m}$ ) induced in the retina in the longitudinal $(\xi)$ and azimuthal $(\phi)$ directions respectively. The variable $k$ is the elastic constant $(\mathrm{N} / \mathrm{m})$ and we have used the notation $\equiv \mathrm{d} / \mathrm{d} \xi$. 
The experimental situation (see Fig. 1) corresponds to the boundary conditions:

$$
\begin{array}{ll}
p\left(r_{0}\right)=0 & \left(\text { retina clamped at inner boundary } r_{0}\right) \\
p\left(\xi_{\mathrm{f}}\right)=\xi_{\mathrm{f}}-r_{1} & \text { (retina clamped at outer boundary } \left.r_{1}\right) \\
r^{\prime}\left(r_{0}\right)=r_{0} & \left(\text { no displacement where clamped at } r_{0}\right) \\
r^{\prime}\left(\xi_{f}\right)=r_{1} & \text { (no displacement where clamped at } \left.r_{1}\right) \\
z\left(\xi_{f}\right)=0 & \text { (no vertical displacement at } \left.r_{1}\right)
\end{array}
$$

where $\xi_{f}$ is the value of $\xi$ at $r_{1}$. These equations can now be solved numerically using the NAG routine D02HAF (available from Numerical Algorithms Group Ltd., Wilkinson House, Jordan Hill Road, Oxford OX2 8DR, UK). Fig. 3 shows typical calculated retinal profiles $z\left(r^{\prime}\right) / r^{\prime}$, rendered into three dimensions.

\section{RESULTS}

In order to solve the equations (1) to (5), subject to the above boundary conditions, we need to supply a value for the elastic constant $k$. The equations will then produce a retinal profile, and, in particular, give $z\left(r_{0}\right)$ and $f$, the applied force. These are the two quantities measured in the bovine retina experiments, so we can vary $k$ and $\xi_{f}$ such that the equations produce the observed values for $z\left(r_{0}\right)$ and $f$. For example, the following data:

$\begin{array}{lccc}f(\mathrm{mN}) & z\left(r_{0}\right)(\mathrm{mm}) & r_{0}(\mathrm{~mm}) & r_{1}(\mathrm{~mm}) \\ 7.35 & 6.0 & 0.5 & 7.5\end{array}$

could be fitted by using the values $\xi_{\mathrm{f}}=10.05 \mathrm{~mm}$ and $k=2.28 \mathrm{~N} / \mathrm{m}$. Using this calculated value of $k$, we can now attempt to predict what values of $f$ should have produced a given $z\left(r_{0}\right)$ by varying $\xi_{f}$ until the correct $z\left(r_{0}\right)$ is obtained. In the following table, $f_{\mathrm{a}}$ is the experimentally applied force, $f_{\mathrm{c}}$ is the calculated value, $f_{\mathrm{d}}$ is the difference in these values and $f_{\%}$ is the value of the difference as a percentage of $f_{\mathrm{a}}$. Distances are measured in millimetres and forces in millinewtons.

$\begin{array}{lcccccr}r_{0} & r_{1} & \xi_{\mathrm{f}} & f_{\mathrm{c}} & f_{\mathrm{a}} & f_{\mathrm{d}} & f_{\%} \\ 0.5 & 9.5 & 10.1 & 3.41 & 4.90 & 1.49 & 37 \\ 0.5 & 3.5 & 5.85 & 9.04 & 9.81 & 0.77 & 7\end{array}$

As can be seen from the table, the value of $k$ of $2.28 \mathrm{~N} / \mathrm{m}$ gives reasonable fits to the experimental data. If we take $k$ to be approximately $2 \mathrm{~N} / \mathrm{m}$ and if the retinal thickness peripheral to the macula is taken to be about $0.1 \mathrm{~mm}$, this gives a Young's modulus $E$ of approximately $2 \times 10^{4} \mathrm{~Pa}$, i.e. about 100 times weaker than a sheet of typical rubber of the same thickness ${ }^{10}$ for which $E \approx 10^{6} \mathrm{~Pa}$. This is in good agreement with $\mathrm{Wu}$ et $a l^{5}$ who measured various moduli by stretching small strips of bovine retina.

\section{DISCUSSION}

We do not claim that great numerical accuracy should be attributed to the calculated value of Young's modulus, since the real retina, although possessing elastic properties, is not a homogeneous structure. The presence and direction of blood vessels and localised weak regions affect retinal strength and elasticity. The experiments were designed to show in a qualitative way that the retina has elastic properties, and although we have calculated a value for Young's modulus this result must be viewed in the context of the true retinal structure. Accurate measurements of height $z\left(r_{0}\right)$ and applied force $f$ are difficult to obtain in a consistent way due to this inhomogeneity of the retinal material.

In this paper we have used a more realistic model for weak incompressible solids than that previously reported ${ }^{6}$ which uses a Poisson ratio $\sigma=1 / 2$ (volume-preserving distortions.) We have taken data from the in vitro stretching of bovine retina, and have fitted those data to the mathematical model. In doing so we have obtained an estimate for Young's modulus of bovine retina of approximately $2 \times 10^{4} \mathrm{~Pa}$. This value can now be used in the model to determine other effects which occur during stretching of detached retina, such as the use of heavy liquids in epiretinal membrane peeling and retinal tearing.

Key words: Elasticity, Mathematical modelling, Retina, Young's modulus.

\section{REFERENCES}

1. Schepens CL: Retinal Detachment. Philadelphia: Saunders, 1983: 694-742.

2. Zauberman H, Berman ER: Measurement of adhesive forces between the sensory retina and the pigment epithelium. Exp Eye Res 1969, 8: 276-83.

3. Zauberman H, de Guillebon H, Holly FJ: Retinal traction in vitro: biophysical aspects. Invest Ophthalmol 1972, 11: 46-55.

4. de Guillebon $\mathrm{H}$, Zauberman $\mathrm{H}$ : Experimental retinal detachment: biophysical aspects of retinal peeling and stretching. Arch Ophthamol 1972, 87: 545-8.

5. Wu W, Peters WH, Hammer ME: Basic mechanical properties of retina in simple elongation. J Biomech Eng 1987, 109: 65-7.

6. Stevens JD, Jones IL, Warner M, Lavin M, Lever P: Mathematical modelling of retinal tear formation: implications for the use of heavy liquids. Eye 1992, 6: 69-74.

7. Moses RA, Hart WM Jr, (eds): Adler's Physiology of the Eye: Clinical Application, 8th edn. St. Louis: CV Mosby, 1987, 458-90.

8. Ryan SJ: Retina, vol 1, St. Louis: CV Mosby, 1989, 13-16.

9. Kain HL: A new model for examining chorioretinal adhesion experimentally. Arch Ophthalmol 1984, 102: 608-11.

10. Harrison RD (ed.): Book of Data. London: Longman Group, 1977. 